\title{
Review of: "Psychosocial stress and cortisol stress reactivity predict breast milk composition"
}

Jing $\mathrm{Li}^{1}$

1 Nanchang University

Potential competing interests: The author(s) declared that no potential competing interests exist.

The authors studied a large number of Polish breastfeeding mothers under maternal perinatal stress. The six-month Recent Life Change Questionnaire was used to evaluated the degree of stress. At the same time, the reactivity of stress was measured by cold pressor test and cortisol content. The human milk was collected to analyze the content of protein, fatty acids and other components. By monitoring the cortisol content, the authors then analyzed the association between stress reactivity and the composition of breast milk. The authors had found that the stress reactivity and psychosocial stress all affected the quality of human breast milk. The study provided a relatively novel perspective for understanding the relationship between the psychological status and composition of human milk. However, I have difficulties to judge the statistical part of the manuscript and hope another reviewer can deal with this. I recommend publication after the authors have focused on the following concerns:

1. It is unclear how the cold pressor test and monitoring cortisol can reflect the degree of stress reactivity. Please write in your introduction about this in details.

2. I understand the short-term effect of cortisol. But are there other influence factors to change the composition of human milk?

3. I recommend the authors think of the title. It is not clear why 'psychosocial stress and cortisol stress reactivity' can 'predict' the milk composition.

4. Abstract: The authors need to mention the grouping principle.

5. The authors should discuss the limitations of the design of experiments.

6. In table 2, I recommend to add the $\mathrm{p}$ value.

7. In table 5, the authors need to explain the meaning of model 0 and model1.

8. The authors should decrease the usage of ' $(R L C)$ '. You use too much '( $R L C)$ ' to emphasize the maternal stress. 